\title{
Die Behandlung stressinduzierter Schmerzen im klinischen Alltag
}

Die in diesem Heft publizierte Arbeit von Egle et al. [1] befasst sich mit der detaillierten Darstellung der neueren Erkenntnisse stressinduzierter Schmerzzustände. Mit einem kurzen Rückgriff auf das von Engels entwickelte bio-psycho-soziale Konzept, das unser Medizinverständnis vor vielen Jahren revolutioniert hat, werden die Resultate der Hirnforschung der letzten 15 Jahre in dieses Konzept eingefügt und damit die bislang unscharfen Vorstellungen von den zugrundeliegenden epigenetischen, psychoneuroendokrinologischen und psychoneuroimmunologischen Mechanismen geklärt und erläutert.

Durch diese Erkenntnisse wird einmal mehr klar, dass es sich bei chronischen Schmerzzuständen ohne nachweisbare Organschädigung nicht um eingebildete Schmerzen handelt oder diese nur Ausdruck einer anderen psychischen Erkrankung wie Depression oder Angst sind, sondern eine eigene Entität darstellen. Damit wird der Beigeschmack der Simulierung bei den erwähnten Diagnosen wie Fibromyalgie-Syndrom, LWS-Schmerzen u.a. klar widerlegt.

Der kurze Abriss über die Notwendigkeit der differenzierten Schmerzdiagnostik und die daraus resultierenden Überlegungen für die therapeutischen Ansätze schafft Klarheit, dass erst nach Klärung der Pathogenese betreffend nozizeptiver, neuropathischer oder gemischter Schmerzen die Diagnose einer stressinduzierten Schmerzstörung gestellt werden darf, wobei auch chronische Schmerzzustände aufgrund von Organ- oder Gewebeschäden mit psychischen Begleitreaktionen einhergehen können.

Egle zeigt die grosse Bedeutung der biografischen Entwicklung für das Auftreten von chronischen, stressbedingten Schmerzzuständen auf, ebenso die Entwicklung von Resilienzfaktoren, ohne dabei in einen therapeutischen Pessimismus zu verfallen. Das klare Statement der Behandelbarkeit dieses Symptomkomplexes ist eine wichtige positive Botschaft und schützt vor Nihilismus, ohne die Komplexität der Erkrankung und notabene der Therapie zu verschweigen.
Eine wichtige Erkenntnis ist die Unwirksamkeit pharmakologischer Interventionen. Auf dem Hintergrund der Opiatepidemie in den USA ist diese klare Stellungnahme essentiell. Betreffend Therapie wird klar dargestellt, dass zu Beginn eine stationäre Behandlung häufig nicht zu umgehen ist. Daher stellen die in der vorliegenden Arbeit anschaulich dargestellten Zusammenhänge auch ein gutes Argumentarium in der Kommunikation mit den Kostenträgern für das Erteilen einer Kostengutsprache für den stationären Aufenthalt in einer entsprechenden Institution dar. Das multimodale Konzept mit Elementen der Edukation, der Arzt-Patient-Beziehung, der Reflexion und der Veränderung eingefahrener Muster überzeugt. Ganz wichtig erscheint der Hinweis über die Notwendigkeit eines Analgetika-Entzuges sowie des Erfassens konkommittierender psychischer Störungen, vor allem der Angststörung. Ebenso kommen mehr oder weniger aktive körperbezogene Verfahren zum Einsatz.

Für spezialisierte Therapeuten, die in diesem herausfordernden Feld tätigt sind, aber auch für Internisten und Hausärzte, bietet die vorliegende Arbeit eine hervorragende Zusammenfassung der heute vorliegenden wissenschaftlichen Erkenntnisse und der möglichen Therapiestrategien.

\section{Bibliografie}

1. Egle UT, Seeher C, Cattapan K: Bio-psycho-soziale Therapie bei stressinduzierten Schmerzzuständen. Praxis 2020; 109: 259-263.

KD Dr. med. Christel Nigg

Chefärztin Klinik Susenberg

Schreberweg 9

8044 Zürich

c.nigg@susenbergklinik.ch 